\title{
Hybrid Textures of the Right-Handed Majorana Neutrino Mass Matrix
}

\author{
S. Dev ${ }^{* a, b}$, Radha Raman Gautam ${ }^{\dagger a}$ and Lal Singh ${ }^{\ddagger a}$ \\ ${ }^{a}$ Department of Physics, Himachal Pradesh University, Shimla 171005, INDIA. \\ ${ }^{b}$ Department of Physics, School of Sciences, HNBG Central University, Srinagar, \\ Uttarakhand 246174, INDIA.
}

\begin{abstract}
We perform a systematic study of neutrino mass matrices having a vanishing cofactor and an equality between two cofactors of the mass matrix. Such texture structures of the effective neutrino mass matrix arise from type-I seesaw mechanism when the Dirac neutrino mass matrix is diagonal with two equal elements and the right-handed Majorana neutrino mass matrix has hybrid textures with one equality of matrix elements and one zero matrix element. For three righthanded neutrinos there are sixty possible hybrid textures out of which only six are excluded by the present experimental data. We show that such textures can be derived using discrete symmetries. The predictions of experimentally allowed textures are examined for unknown parameters such as the effective Majorana mass of the electron neutrino and the Dirac-type CP-violating phase.
\end{abstract}

\section{Introduction}

During the past decade various neutrino oscillation experiments have determined the neutrino mass squared differences and the lepton mixing angles with a good precision [1]. Especially the reactor mixing angle $\left(\theta_{13}\right)$ has been measured rather precisely in recent experiments [2, 3, 4, 5, 6]. The best fit value for $\theta_{13}$ is around $9^{\circ}$ which has a large deviation from zero and provides an opportunity for the measurement of Dirac-type CP-violating phase $(\delta)$ in the lepton mixing matrix. Several ideas have been mooted to accommodate the observed pattern of neutrino mass squared differences and mixing angles which include tribimaximal (TBM) mixing [7] where one obtains fixed values of mixing angles and the neutrino masses are independent of the mixing angles. Such textures have been called mass independent textures [8]. On the other hand, zero textures [9, 10] and vanishing minors [11, 12] which relate neutrino masses with mixing angles are called mass-dependent textures. Hybrid textures which imply one equality between matrix elements and one zero element in the effective neutrino mass matrix also fall in the category of mass dependent textures and have been studied earlier in a

\footnotetext{
*dev5703@yahoo.com

†'gautamrrg@gmail.com

†lalsingh96@yahoo.com
} 
basis where the charged lepton mass matrix is diagonal [13, 14, 15] and in a basis where both the charged lepton and the neutrino mass matrix have hybrid textures [16]. In the present work, we examine the phenomenological implications of hybrid textures of the inverse neutrino mass matrix in a basis where the charged lepton mass matrix is diagonal.

To understand the smallness of neutrino masses as compared to charged fermion masses, the seesaw mechanism [17] is regarded as the prime candidate. In the framework of type-I seesaw mechanism, the effective Majorana neutrino mass matrix is given by

$$
M_{\nu} \approx-M_{D} M_{R}^{-1} M_{D}^{T}
$$

where $M_{D}$ is the Dirac neutrino mass matrix and $M_{R}$ is the right-handed Majorana neutrino mass matrix. Within this framework, $M_{\nu}$ is a quantity derived from $M_{D}$ and $M_{R}$. Thus, in the context of type-I seesaw mechanism, the zeros and equalities of $M_{D}$ and $M_{R}$ are more fundamental. In a basis where $M_{D}$ is diagonal, the zero textures of $M_{R}$ show as vanishing cofactors in $M_{\nu}$ [11] and the equalities in $M_{R}$ propagate as equal cofactors in $M_{\nu}$ provided that there are equal non-zero elements in diagonal $M_{D}$ [18. We consider another possibility where $M_{R}$ has hybrid textures in a basis where $M_{D}$ is diagonal with one equality between diagonal elements. Such $M_{D}$ and $M_{R}$ give rise to the neutrino mass matrix having one equality between the cofactors and one vanishing cofactor. The equality between the cofactors and the vanishing cofactor in $M_{\nu}$ correspond to the equal and zero elements of $M_{R}$, respectively. Such textures can also be seen as hybrid textures of the inverse $M_{\nu}$. Thus, effectively we are studying the hybrid textures of $M_{\nu}^{-1}$. For three right-handed neutrinos, there are sixty possible hybrid textures of $M_{R}$ which are listed in Table 1 . To illustrate how such textures can be realised we present a flavor model for class $I A$ using discrete flavor symmetries.

\section{The Neutrino Mass Matrix}

Assuming neutrinos to be Majorana particles, we reconstruct the neutrino mass matrix in the flavor basis (where the charged lepton mass matrix " $M_{l}$ " is diagonal). In this basis, the complex symmetric neutrino mass matrix is diagonalized by a unitary matrix $V^{\prime}$ as

$$
M_{\nu}=V^{\prime} M_{\nu}^{\text {diag }} V^{\prime T}
$$

where $M_{\nu}^{\text {diag }}=\operatorname{diag}\left(m_{1}, m_{2}, m_{3}\right)$.

The unitary matrix $V^{\prime}$ can be parametrized as

$$
V^{\prime}=P_{l} V \quad \text { with } \quad V=U P_{\nu}
$$

where [19]

$$
U=\left(\begin{array}{ccc}
c_{12} c_{13} & s_{12} c_{13} & s_{13} e^{-i \delta} \\
-s_{12} c_{23}-c_{12} s_{23} s_{13} e^{i \delta} & c_{12} c_{23}-s_{12} s_{23} s_{13} e^{i \delta} & s_{23} c_{13} \\
s_{12} s_{23}-c_{12} c_{23} s_{13} e^{i \delta} & -c_{12} s_{23}-s_{12} c_{23} s_{13} e^{i \delta} & c_{23} c_{13}
\end{array}\right)
$$

with $s_{i j}=\sin \theta_{i j}$ and $c_{i j}=\cos \theta_{i j}$ and

$$
P_{\nu}=\left(\begin{array}{ccc}
1 & 0 & 0 \\
0 & e^{i \alpha} & 0 \\
0 & 0 & e^{i(\beta+\delta)}
\end{array}\right), \quad P_{l}=\left(\begin{array}{ccc}
e^{i \varphi_{e}} & 0 & 0 \\
0 & e^{i \varphi_{\mu}} & 0 \\
0 & 0 & e^{i \varphi_{\tau}}
\end{array}\right)
$$




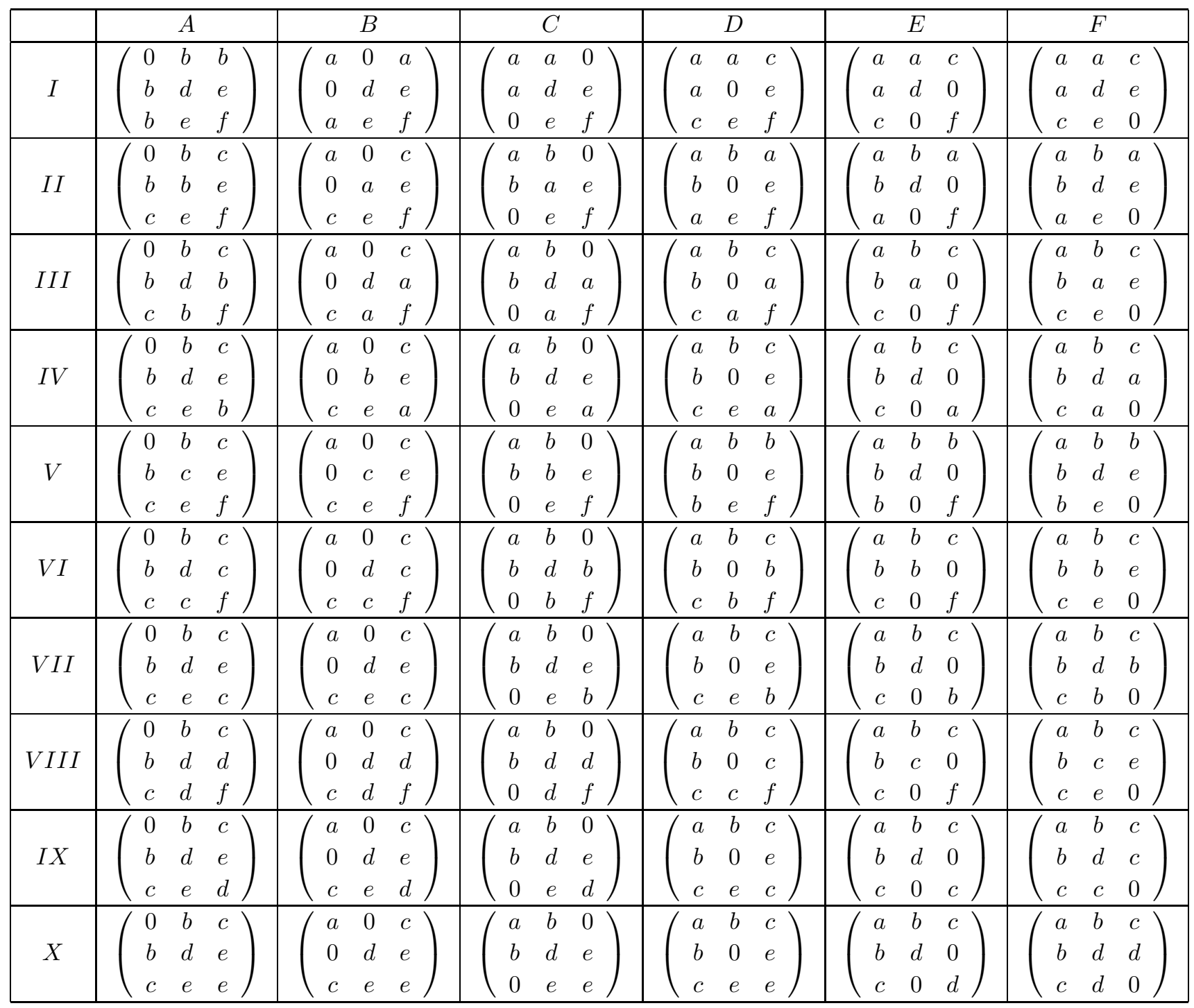

Table 1: Sixty possible hybrid texture structures of $M_{R}$. 
$P_{\nu}$ is the diagonal phase matrix with two Majorana-type CP-violating phases $\alpha, \beta$ and one Diractype CP-violating phase $\delta$. The phase matrix $P_{l}$ is not observable and depends on the phase convention. The matrix $V$ is called the neutrino mixing matrix or the Pontecorvo-Maki-Nakagawa-Sakata (PMNS) matrix [20]. Using Eq. (2) and Eq. (3), the neutrino mass matrix can be written as

$$
M_{\nu}=P_{l} U P_{\nu} M_{\nu}^{\text {diag }} P_{\nu}^{T} U^{T} P_{l}^{T} .
$$

The CP-violation in neutrino oscillation experiments can be described through a rephasing invariant quantity, $J_{C P}$ [21] with $J_{C P}=\operatorname{Im}\left(U_{e 1} U_{\mu 2} U_{e 2}^{*} U_{\mu 1}^{*}\right)$. In the above parametrization, $J_{C P}$ is given by

$$
J_{C P}=s_{12} s_{23} s_{13} c_{12} c_{23} c_{13}^{2} \sin \delta .
$$

\subsection{Neutrino Mass Matrices with an Equality Between the Cofactors and a Van- ishing Cofactor}

In the effective neutrino mass matrix, the simultaneous existence of an equality between two cofactors and one vanishing cofactor implies

$$
\begin{aligned}
& \left(-1^{(\gamma \xi)}\right)\left(e^{i\left(\varphi_{a}+\varphi_{b}+\varphi_{c}+\varphi_{d}\right)} M_{\nu(a b)} M_{\nu(c d)}-e^{i\left(\varphi_{f}+\varphi_{g}+\varphi_{m}+\varphi_{n}\right)} M_{\nu(f g)} M_{\nu(m n)}\right)- \\
& \left(-1^{(\zeta \eta)}\right)\left(e^{i\left(\varphi_{p}+\varphi_{q}+\varphi_{r}+\varphi_{s}\right)} M_{\nu(p q)} M_{\nu(r s)}-e^{i\left(\varphi_{t}+\varphi_{u}+\varphi_{v}+\varphi_{w}\right)} M_{\nu(t u)} M_{\nu(v w)}\right)=0 \\
& e^{i\left(\varphi_{a^{\prime}}+\varphi_{b^{\prime}}+\varphi_{c^{\prime}}+\varphi_{d^{\prime}}\right)} M_{\nu\left(a^{\prime} b^{\prime}\right)} M_{\nu\left(c^{\prime} d^{\prime}\right)}-e^{i\left(\varphi_{f^{\prime}}+\varphi_{g^{\prime}}+\varphi_{m^{\prime}}+\varphi_{n^{\prime}}\right)} M_{\nu\left(f^{\prime} g^{\prime}\right)} M_{\nu\left(m^{\prime} n^{\prime}\right)}=0 .
\end{aligned}
$$

The condition for equality between two cofactors in the neutrino mass matrix [Eq. (7)] can be written as

$$
\begin{aligned}
& \left(-1^{(\gamma \xi)}\right)\left(Q_{1} M_{\nu(a b)} M_{\nu(c d)}-Q_{2} M_{\nu(f g)} M_{\nu(m n)}\right)- \\
& \left(-1^{(\zeta \eta)}\right)\left(Q_{3} M_{\nu(p q)} M_{\nu(r s)}-Q_{4} M_{\nu(t u)} M_{\nu(v w)}\right)=0 .
\end{aligned}
$$

It is inherent in the properties of cofactors that when we substitute $\varphi_{j},(j=e, \mu, \tau)$, then $Q_{1}=Q_{2}$ and $Q_{3}=Q_{4}$. Thus, we have

$$
\begin{aligned}
& \left(-1^{(\gamma \xi)}\right) Q_{1}\left(M_{\nu(a b)} M_{\nu(c d)}-M_{\nu(f g)} M_{\nu(m n)}\right)- \\
& \left(-1^{(\zeta \eta)}\right) Q_{3}\left(M_{\nu(p q)} M_{\nu(r s)}-M_{\nu(t u)} M_{\nu(v w)}\right)=0 .
\end{aligned}
$$

or

$$
\begin{aligned}
& \left(-1^{(\gamma \xi)}\right) Q\left(M_{\nu(a b)} M_{\nu(c d)}-M_{\nu(f g)} M_{\nu(m n)}\right)- \\
& \left(-1^{(\zeta \eta)}\right)\left(M_{\nu(p q)} M_{\nu(r s)}-M_{\nu(t u)} M_{\nu(v w)}\right)=0 .
\end{aligned}
$$

where $Q=\frac{Q_{1}}{Q_{3}}$.

The two conditions Eq. (8) and Eq. (11) take the following form when expressed in terms of the mixing matrix elements and mass eigenvalues:

$$
\begin{aligned}
& \sum_{k, l=1}^{3}\left\{\left(-1^{(\gamma \xi)}\right) Q\left(V_{a k} V_{b k} V_{c l} V_{d l}-V_{f k} V_{g k} V_{m l} V_{n l}\right)-\right. \\
& \left.\quad\left(-1^{(\zeta \eta)}\right)\left(V_{p k} V_{q k} V_{r l} V_{s l}-V_{t k} V_{u k} V_{v l} V_{w l}\right)\right\} m_{k} m_{l}=0, \\
& \sum_{k, l=1}^{3}\left(V_{a^{\prime} k} V_{b^{\prime} k} V_{c^{\prime} l} V_{d^{\prime} l}-V_{f^{\prime} k} V_{g^{\prime} k} V_{m^{\prime} l} V_{n^{\prime} l}\right) m_{k} m_{l}=0 .
\end{aligned}
$$


The above equations can be rewritten as

$$
\begin{aligned}
& m_{1} m_{2} A_{3} e^{2 i \alpha}+m_{2} m_{3} A_{1} e^{2 i(\alpha+\beta+\delta)}+m_{3} m_{1} A_{2} e^{2 i(\beta+\delta)}=0, \\
& m_{1} m_{2} B_{3} e^{2 i \alpha}+m_{2} m_{3} B_{1} e^{2 i(\alpha+\beta+\delta)}+m_{3} m_{1} B_{2} e^{2 i(\beta+\delta)}=0
\end{aligned}
$$

where

$$
\begin{aligned}
& A_{h}=\left(-1^{(\gamma \xi)}\right) Q\left(U_{a k} U_{b k} U_{c l} U_{d l}-U_{f k} U_{g k} U_{m l} U_{n l}\right)- \\
& \quad\left(-1^{(\zeta \eta)}\right)\left(U_{p k} U_{q k} U_{r l} U_{s l}-U_{t k} U_{u k} U_{v l} U_{w l}\right)+(k \leftrightarrow l), \\
& B_{h}=\left(U_{a^{\prime} k} U_{b^{\prime} k} U_{c^{\prime} l} U_{d^{\prime} l}-U_{f^{\prime} k} U_{g^{\prime} k} U_{m^{\prime} l} U_{n^{\prime} l}\right)+(k \leftrightarrow l)
\end{aligned}
$$

with $(h, k, l)$ as the cyclic permutation of $(1,2,3)$. The two complex Eqs. (14) and (15) contain nine physical parameters which are the three neutrino masses $\left(m_{1}, m_{2}, m_{3}\right)$, the three mixing angles $\left(\theta_{12}, \theta_{23}, \theta_{13}\right)$ and three $\mathrm{CP}$-violating phases $(\alpha, \beta, \delta)$. In addition, there are three unobservable phases $\left(\varphi_{e}, \varphi_{\mu}, \varphi_{\tau}\right)$ which enter in the mass ratios as a phase difference. The masses $m_{2}$ and $m_{3}$ can be calculated from the mass-squared differences $\Delta m_{21}^{2}\left(\Delta m_{21}^{2} \equiv m_{2}^{2}-m_{1}^{2}\right)$ and $\left|\Delta m_{32}^{2}\right|$ using the following relations

$$
m_{2}=\sqrt{m_{1}^{2}+\Delta m_{21}^{2}}, \quad m_{3}=\sqrt{m_{2}^{2}+\left|\Delta m_{32}^{2}\right|}
$$

with $m_{2}>m_{3}$ for an Inverted Spectrum (IS) and $m_{2}<m_{3}$ for the Normal Spectrum (NS). By using the experimental inputs of the two mass-squared differences and the three mixing angles, we can constrain the other parameters. Simultaneously solving Eqs. (14) and (15) for the two mass ratios, we obtain

$$
\begin{aligned}
& \frac{m_{1}}{m_{2}} e^{-2 i \alpha}=\frac{A_{3} B_{2}-A_{2} B_{3}}{A_{1} B_{3}-A_{3} B_{1}}, \\
& \frac{m_{1}}{m_{3}} e^{-2 i \beta}=\frac{A_{2} B_{3}-A_{3} B_{2}}{A_{1} B_{2}-A_{2} B_{1}} e^{2 i \delta} .
\end{aligned}
$$

We denote the magnitudes of the above two mass ratios by

$$
\begin{aligned}
\rho & =\left|\frac{m_{1}}{m_{3}} e^{-2 i \beta}\right|, \\
\sigma & =\left|\frac{m_{1}}{m_{2}} e^{-2 i \alpha}\right| .
\end{aligned}
$$

The Majorana-type CP-violating phases $\alpha$ and $\beta$ are given by

$$
\begin{aligned}
\alpha & =-\frac{1}{2} \arg \left(\frac{A_{3} B_{2}-A_{2} B_{3}}{A_{1} B_{3}-A_{3} B_{1}}\right), \\
\beta & =-\frac{1}{2} \arg \left(\frac{A_{2} B_{3}-A_{3} B_{2}}{A_{1} B_{2}-A_{2} B_{1}} e^{2 i \delta}\right) .
\end{aligned}
$$

Since, $\Delta m_{21}^{2}$ and $\left|\Delta m_{32}^{2}\right|$ are experimentally known, the two mass ratios $(\rho, \sigma)$ in Eqs. (21) and (22) can be used to calculate $m_{1}$. This can be done by inverting Eq. (18) to obtain the two values of $m_{1}$, viz.

$$
m_{1}=\sigma \sqrt{\frac{\Delta m_{21}^{2}}{1-\sigma^{2}}}, \quad m_{1}=\rho \sqrt{\frac{\Delta m_{21}^{2}+\left|\Delta m_{32}^{2}\right|}{1-\rho^{2}}} .
$$


There exists a permutation symmetry between different patterns of two-zero textures [10], in the case of two vanishing minors [12] and in the case of two equalities in $M_{\nu}$ [18. Similarly, there exists a permutation symmetry between different hybrid textures of $M_{\nu}^{-1}$. The permutation matrix is given by

$$
P_{23}=\left(\begin{array}{lll}
1 & 0 & 0 \\
0 & 0 & 1 \\
0 & 1 & 0
\end{array}\right) .
$$

For example, the neutrino mass matrix for class $I C$ can be obtained from class $I B$ by the transformation

$$
M_{\nu}^{I C}=P_{23} M_{\nu}^{I B} P_{23}^{T} .
$$

This leads to the following relations between the parameters of the classes related by the 2-3 permutation symmetry:

$$
\theta_{12}^{I C}=\theta_{12}^{I B}, \theta_{13}^{I C}=\theta_{13}^{I B}, \theta_{23}^{I C}=\frac{\pi}{2}-\theta_{23}^{I B}, \delta^{I C}=\delta^{I B}-\pi .
$$

The textures related by the 2-3 permutation symmetry are:

$$
\begin{aligned}
& I B \leftrightarrow I C, I D \leftrightarrow I I F, I E \leftrightarrow I I E, I F \leftrightarrow I I D, I I A \leftrightarrow V I I A, I I B \leftrightarrow I V C, I I C \leftrightarrow I V B, \\
& I I I A \leftrightarrow V I A, I I I B \leftrightarrow I I I C, I I I D \leftrightarrow I V F, I I I E \leftrightarrow I V E, I I I F \leftrightarrow I V D, I V A \leftrightarrow V A, \\
& V B \leftrightarrow V I I C, V C \leftrightarrow V I I B, V D \leftrightarrow V F, V I B \leftrightarrow V I C, V I D \leftrightarrow I X F, V I E \leftrightarrow I X E, \\
& V I F \leftrightarrow I X D, V I I D \leftrightarrow V I I I F, V I I E \leftrightarrow V I I I E, V I I F \leftrightarrow V I I I D, V I I I A \leftrightarrow X A, \\
& V I I I B \leftrightarrow X C, V I I I C \leftrightarrow X B, I X B \leftrightarrow I X C, X D \leftrightarrow X F .
\end{aligned}
$$

The remaining textures namely:

$$
I A, V E, I X A, X E
$$

transform unto themselves.

\section{Numerical Analysis and Results}

The latest global fit results on neutrino oscillation parameters at $1 \sigma, 2 \sigma$ and $3 \sigma$ [22] are summarised in Table 2. The effective Majorana mass of the electron neutrino $\left(M_{e e}\right)$ which determines the rate of neutrinoless double beta (NDB) decay is given by

$$
M_{e e}=\left|m_{1} c_{12}^{2} c_{13}^{2}+m_{2} s_{12}^{2} c_{13}^{2} e^{2 i \alpha}+m_{3} s_{13}^{2} e^{2 i \beta}\right| .
$$

Observation of NDB decay will imply that neutrinos are Majorana fermions. NDB decay also provides a way to probe the neutrino mass scale. There are a large number of projects such as CUORICINO [23], CUORE [24], GERDA [25], MAJORANA [26], SuperNEMO [27], EXO [28], GENIUS [29] which aim to achieve a sensitivity upto $0.01 \mathrm{eV}$ for $M_{e e}$. We take the upper limit of $M_{e e}$ to be $0.5 \mathrm{eV}$ [30]. In addition, cosmological observations put an upper bound on the sum of light neutrino masses

$$
\Sigma=\sum_{i=1}^{3} m_{i} .
$$




\begin{tabular}{|c|c|}
\hline Parameter & $\begin{array}{l}\text { Mean } \begin{array}{l}(+1 \sigma,+2 \sigma,+3 \sigma) \\
(-1 \sigma,-2 \sigma,-3 \sigma)\end{array} \\
\end{array}$ \\
\hline$\Delta m_{21}^{2}\left[10^{-5} \mathrm{eV}^{2}\right]$ & $7.62_{(-0.19,-0.35,-0.5)}^{(+0.19,+0.39,+0.58)}$ \\
\hline$\Delta m_{31}^{2}\left[10^{-3} e V^{2}\right]$ & $\begin{array}{c}2.55_{(-0.06,+0.13,+0.19)}^{(+0.09,-0.19,-0.24)} \\
\left(-2.43_{(-0.07,-0.15,-0.21)}^{(+0.09,+0.19,+0.24)}\right) \\
\end{array}$ \\
\hline $\sin ^{2} \theta_{12}$ & $0.32_{(-0.017,-0.03,-0.05)}^{(+0.016,+0.03,+0.05)}$ \\
\hline $\sin ^{2} \theta_{23}$ & $\begin{array}{c}0.613_{(-0.022,-0.047,+0.067)}^{(+0.023,-0.25)}, \\
\left(0.60_{(-0.026,+0.05,+0.07)}^{(+0.026,+0.0210,-0.230)}\right)\end{array}$ \\
\hline $\sin ^{2} \theta_{13}$ & $\begin{array}{c}0.0246_{(-0.0028,+0.0056,+0.0076)}^{(+0.0029,-0054,-0.0084)}, \\
\left(0.0250_{(-0.0026,+0.005,+0.008)}^{(+0.0026,+0.005,-0.008)}\right)\end{array}$ \\
\hline
\end{tabular}

Table 2: Current Neutrino oscillation parameters from global fits [22. The upper (lower) row corresponds to Normal (Inverted) Spectrum, with $\Delta m_{31}^{2}>0\left(\Delta m_{31}^{2}<0\right)$.

Recent data from Planck satellite [31] combined with other cosmological data limit $\Sigma<0.23$ at $95 \%$ Confidence level. However, these bounds are strongly dependent on model details and the data set used. Thus, in our numerical analysis we take the upper limit on $\Sigma$ to be $1 \mathrm{eV}$.

In our numerical programs, the constraints implied by an equality between the two cofactors of $M_{\nu}$ and one vanishing cofactor in $M_{\nu}$ are used by equating the two values of $m_{1}$ obtained in Eq. (25). These two values of $m_{1}$ should be equal to within the errors of the oscillation parameters. We vary the known oscillation parameters randomly within their $3 \sigma$ experimental ranges given in [22]. The unknown Dirac-type CP-violating phase $\delta$ is varied randomly within its full possible range. For the numerical analysis, we generate $10^{7}$ random points $\left(10^{8}\right.$ when the number of allowed points is small) for the allowed $3 \sigma$ ranges of oscillation parameters.

Main results of the numerical analysis are:

- Six textures, viz., II A, IIIA, IVA, VA,VIA and VIIA are excluded by the present experimental data.

- Textures IE, IIE, IIID, IIIE, IVE, IVF, VIE, VIIE, VIIF, VIIID,VIIIE and IXE lead to a normal spectrum only.

- Textures $I A, I B, I C, I I C, I I I B, I I I C, I V B, V I I I A, I X A$ and $X A$ lead to an inverted spectrum only.

- The allowed points for the following textures are very few for $I B, I F, I I D, I I F, I I I F, I V D, V E, V I D, V I I I A, I X A, I X F, X D, X E, X F$ for an inverted spectrum

and $I X B, I X C$ for both inverted and normal spectra.

We have generated $10^{8}$ random points for these textures.

- All the viable textures except IA, IB, IC, VIIE, VIIIA, VIIIE and XA allow quasidegenerate spectrum.

- Many of the classes predict a constrained range for $M_{e e}$. 


\begin{tabular}{|c|c|c|c|c|c|}
\hline Texture & Spectrum & $M_{e e}(\mathrm{eV})$ & $m_{o}(\mathrm{eV})$ & $\theta_{23}$ & Majorana Phases \\
\hline IA & IS & $0.01-0.055$ & 0.0007 & - & $\beta=70^{\circ}-110^{\circ}$ \\
\hline IB (IC) & IS & $0.034-0.05$ & 0.007 & - & $\alpha=25^{\circ}-55^{\circ}, 125^{\circ}-155^{\circ}$ \\
\hline ID (IIF) & $\begin{array}{l}\mathrm{NS} \\
\mathrm{IS}\end{array}$ & $\begin{array}{c}0-0.15 \\
0.03-0.20\end{array}$ & $\begin{array}{c}0.003 \\
0.03\end{array}$ & $\begin{array}{c}- \\
<45^{\circ}\left(>45^{\circ}\right)\end{array}$ & $\begin{array}{c}\alpha=50^{\circ}-130^{\circ} \\
\alpha=45^{\circ}-165^{\circ}, 115^{\circ}-135^{\circ}\end{array}$ \\
\hline IE (IIE) & $\mathrm{NS}$ & $0-0.07$ & 0.002 & - & $\alpha=40^{\circ}-140^{\circ}$ \\
\hline$\overline{\mathrm{IF}(\mathrm{IID})}$ & $\begin{array}{l}\mathrm{NS} \\
\mathrm{IS}\end{array}$ & $\begin{array}{c}0-0.16 \\
0.02-0.16\end{array}$ & $\begin{array}{c}0.002 \\
0.06\end{array}$ & $\begin{array}{c}- \\
>45^{\circ}\left(<45^{\circ}\right)\end{array}$ & $\begin{array}{l}\alpha=50^{\circ}-130^{\circ} \\
\alpha=60^{\circ}-120^{\circ}\end{array}$ \\
\hline IIB (IVC) & $\begin{array}{l}\mathrm{NS} \\
\mathrm{IS}\end{array}$ & $\begin{array}{l}0.04-0.35 \\
0.04-0.35\end{array}$ & $\begin{array}{c}0.05 \\
0.008 \\
\end{array}$ & $\begin{array}{l}- \\
-\end{array}$ & $\begin{array}{c}\alpha=0^{\circ}-30^{\circ}, 150^{\circ}-180^{\circ} \\
\alpha, \beta=0^{\circ}-30^{\circ}, 150^{\circ}-180^{\circ}\end{array}$ \\
\hline IIC (IVB) & IS & $0.02-0.35$ & 0.01 & - & $\alpha=0^{\circ}-80^{\circ}, 100^{\circ}-180^{\circ}$ \\
\hline IIIB (IIIC) & IS & $0.03-0.30$ & 0.015 & - & $\alpha=0^{\circ}-60^{\circ}, 120^{\circ}-180^{\circ}$ \\
\hline IIID (IVF) & NS & $0.002-0.25$ & 0.015 & - & - \\
\hline IIIE (IVE) & NS & $0-0.35$ & 0.01 & - & $\alpha=5^{\circ}-175^{\circ}$ \\
\hline IIIF (IVD) & $\begin{array}{l}\mathrm{NS} \\
\mathrm{IS} \\
\end{array}$ & $\begin{array}{c}0-0.16 \\
0.04-0.18 \\
\end{array}$ & $\begin{array}{l}0.01 \\
0.02 \\
\end{array}$ & $>45^{\circ}\left(<45^{\circ}\right)$ & $\begin{array}{c}\alpha=60^{\circ}-120^{\circ} \\
\alpha=0^{\circ}-80^{\circ}, 100^{\circ}-180^{\circ}\end{array}$ \\
\hline VB (VIIC) & $\begin{array}{l}\mathrm{NS} \\
\mathrm{IS}\end{array}$ & $\begin{array}{c}0.004-0.35 \\
0.04-0.35\end{array}$ & $\begin{array}{l}0.005 \\
0.010\end{array}$ & $\begin{array}{l}>45^{\circ}\left(<45^{\circ}\right) \\
<45^{\circ}\left(>45^{\circ}\right)\end{array}$ & $\begin{array}{l}\alpha=0^{\circ}-20^{\circ}, 160^{\circ}-180^{\circ} \\
\alpha=0^{\circ}-20^{\circ}, 160^{\circ}-180^{\circ}\end{array}$ \\
\hline $\mathrm{VC}(\mathrm{VIIB})$ & $\begin{array}{l}\mathrm{NS} \\
\mathrm{IS}\end{array}$ & $\begin{array}{c}0.005-0.30 \\
0.01-0.30\end{array}$ & $\begin{array}{c}0.007 \\
0.01\end{array}$ & $\begin{array}{l}>45^{\circ}\left(<45^{\circ}\right) \\
<45^{\circ}\left(>45^{\circ}\right)\end{array}$ & $\begin{array}{c}\alpha=0^{\circ}-10^{\circ}, 170^{\circ}-180^{\circ} \\
-\end{array}$ \\
\hline $\mathrm{VD}(\mathrm{VF})$ & $\begin{array}{l}\text { NS } \\
\text { IS }\end{array}$ & $\begin{array}{c}0-0.35 \\
0.04-0.35\end{array}$ & $\begin{array}{l}0.001 \\
0.020\end{array}$ & $\begin{array}{l}- \\
-\end{array}$ & $\alpha=0^{\circ}-45^{\circ}, 135^{\circ}-180^{\circ}$ \\
\hline $\mathrm{VE}$ & $\begin{array}{l}\mathrm{NS} \\
\mathrm{IS}\end{array}$ & $\begin{array}{c}0-0.20 \\
0.1-0.35\end{array}$ & $\begin{array}{c}0.001 \\
0.10\end{array}$ & $\begin{array}{ll}- \\
-\end{array}$ & $\alpha=0^{\circ}-10^{\circ}, \quad 170^{\circ}-180^{\circ}, \beta=20^{\circ}-160^{\circ}$ \\
\hline VIB (VIC) & $\begin{array}{l}\mathrm{NS} \\
\mathrm{IS} \\
\end{array}$ & $\begin{array}{c}0.002-0.35 \\
0.02-0.35 \\
\end{array}$ & $\begin{array}{l}0.004 \\
0.013\end{array}$ & $\begin{array}{l}- \\
-\end{array}$ & $\begin{array}{l}\alpha=0^{\circ}-20^{\circ}, 160^{\circ}-180^{\circ} \\
\alpha=0^{\circ}-80^{\circ}, 100^{\circ}-180^{\circ}\end{array}$ \\
\hline VID (IXF) & $\begin{array}{l}\text { NS } \\
\text { IS }\end{array}$ & $\begin{array}{c}0.005-0.12 \\
0.03-0.14\end{array}$ & $\begin{array}{c}0.006 \\
0.04\end{array}$ & $\begin{array}{c}- \\
<45^{\circ}\left(>45^{\circ}\right)\end{array}$ & $\begin{array}{c}\alpha=0^{\circ}-80^{\circ}, 100^{\circ}-180^{\circ} \\
\alpha=60^{\circ}-120^{\circ}\end{array}$ \\
\hline VIE (IXE) & $\mathrm{NS}$ & $0.001-0.07$ & 0.016 & - & $\alpha=40^{\circ}-140^{\circ}$ \\
\hline VIF (IXD) & $\begin{array}{l}\mathrm{NS} \\
\text { IS }\end{array}$ & $\begin{array}{c}0.002-0.30 \\
0.05-0.35 \\
\end{array}$ & $\begin{array}{l}0.003 \\
0.044\end{array}$ & $>45^{\circ}\left(<45^{\circ}\right)$ & $\alpha=0^{\circ}-80^{\circ}, 100^{\circ}-180^{\circ}$ \\
\hline VIID (VIIIF) & $\begin{array}{c}\text { NS } \\
\text { IS }\end{array}$ & $\begin{array}{c}0-0.30 \\
0.02-0.30 \\
\end{array}$ & $\begin{array}{c}0.0008 \\
0.045 \\
\end{array}$ & $\begin{array}{l}>45^{\circ}\left(<45^{\circ}\right) \\
<45^{\circ}\left(>45^{\circ}\right)\end{array}$ & - \\
\hline VIIE (VIIIE) & NS & $0.008-0.03$ & 0.02 & - & $\alpha=50^{\circ}-130^{\circ}$ \\
\hline$\overline{\text { VIIF (VIIID) }}$ & $\mathrm{NS}$ & $0.008-0.028$ & 0.007 & - & $\alpha=30^{\circ}-150^{\circ}$ \\
\hline VIIIA (XA) & IS & $0.01-0.05$ & 0.0008 & - & $\beta=70^{\circ}-110^{\circ}$ \\
\hline VIIIB (XC) & $\begin{array}{l}\mathrm{NS} \\
\mathrm{IS}\end{array}$ & $\begin{array}{c}0.003-0.25 \\
0.01-0.25\end{array}$ & $\begin{array}{l}0.005 \\
0.003\end{array}$ & $\begin{array}{l}- \\
-\end{array}$ & $\begin{array}{c}\alpha=0^{\circ}-20^{\circ}, 160^{\circ}-180^{\circ} \\
-\end{array}$ \\
\hline VIIIC (XB) & $\begin{array}{l}\text { NS } \\
\text { IS }\end{array}$ & $\begin{array}{l}0.003-0.25 \\
0.003-0.30\end{array}$ & $\begin{array}{l}0.005 \\
0.003\end{array}$ & $\begin{array}{l}- \\
-\end{array}$ & $\begin{array}{c}\alpha=0^{\circ}-20^{\circ}, 160^{\circ}-180^{\circ} \\
-\end{array}$ \\
\hline IXA & IS & - & - & - & - \\
\hline IXB (IXC) & $\begin{array}{l}\text { NS } \\
\text { IS }\end{array}$ & $\begin{array}{c}0.003-0.30 \\
0.01-0.35 \\
\end{array}$ & $\begin{array}{l}0.0052 \\
0.0065 \\
\end{array}$ & $\begin{array}{l}36^{\circ}-48^{\circ}\left(42^{\circ}-56^{\circ}\right) \\
36^{\circ}-46^{\circ}\left(44^{\circ}-56^{\circ}\right)\end{array}$ & $\begin{array}{c}\alpha=0^{\circ}-30^{\circ}, 150^{\circ}-180^{\circ} \\
-\end{array}$ \\
\hline $\mathrm{XD}(\mathrm{XF})$ & $\begin{array}{l}\text { NS } \\
\text { IS }\end{array}$ & $\begin{array}{c}0.001-0.045 \\
0.03-0.13\end{array}$ & $\begin{array}{c}0.0015 \\
0.022\end{array}$ & $\begin{array}{c}- \\
<45^{\circ}\left(>45^{\circ}\right)\end{array}$ & $\begin{array}{l}\alpha=20^{\circ}-160^{\circ} \\
\alpha=35^{\circ}-145^{\circ}\end{array}$ \\
\hline $\mathrm{XE}$ & $\begin{array}{l}\text { NS } \\
\text { IS }\end{array}$ & $\begin{array}{c}0.001-0.30 \\
-\end{array}$ & $\begin{array}{c}0.002 \\
-\end{array}$ & $\begin{array}{l}- \\
-\end{array}$ & $\begin{array}{l}- \\
-\end{array}$ \\
\hline
\end{tabular}

Table 3: The predictions for the phenomenologically viable textures. $m_{o}$ is the lowest mass scale i.e. lowest value of $m_{1}$ for NS and $m_{3}$ for IS. 
- It is found that the smallest neutrino mass cannot be zero for any of the allowed textures.

- For textures:

$I I B, I I C, I I I B, I I I C, I V B, I V C, V B, V C, V D, V E, V F, V I B, V I C, V I I B, V I I C$, $V I I I B, V I I I C, I X B, I X C, X B, X C$ and $V I F$, a non-vanishing reactor mixing angle is an inherent property since for $\theta_{13}=0$ the solar mass square difference $\left(\Delta m_{21}^{2}\right)$ vanishes i.e. $m_{1}=m_{2}$, which is contrary to the experimental observations.

We have summarised the numerical results for all the classes compatible with the present experimental data in Table 3. Some of the interesting results are plotted in Figs. 1-5. Fig. 1(a) shows the correlation plot between the two Majorana-type phases ( $\alpha$ and $\beta$ ) for class $I A(\mathrm{IS})$. For class $I I B(\mathrm{NS})$, $\delta$ approaches $0^{\circ}$ or $180^{\circ}$ with the increase in $M_{e e}$ [Fig. 1(b)]. Also, for the normal spectrum, $J_{C P}$ cannot be equal to zero [Fig. 1(c)] implying that $\mathrm{CP}$ is necessarily violated. There is an interesting correlation between $\theta_{12}$ and $\theta_{23}$ for class $\operatorname{IIIF(NS)}$ [Fig. 1(d)] where $\theta_{23}$ remains above maximal when $\theta_{12}$ is below $34^{\circ}$. For class $I V D, \theta_{23}$ and $\theta_{13}$ are correlated [Figs. 1(e)(NS), 1(f)(IS)]. In Fig. 2, we have given correlation plots for classes $V B$ and $V C$ which are among the most predictive textures in this analysis. The Majorana phases are restricted to a very small range for class $V B$ (IS) [Fig. 2(a)]. The Dirac-type phase is restricted near $90^{\circ}$ or $270^{\circ}$ [Fig. 2(b)] and $J_{C P}$ is always non-zero [Fig. 2(c)] implying that, for inverted spectrum, this class is necessarily CP-violating. For class $V C(\mathrm{NS}), \theta_{23}$ is always above $45^{\circ}$ [Fig. 2(d)] and the Dirac-type phase is fixed near $90^{\circ}$ and $270^{\circ}$ for $M_{e e}>0.05 \mathrm{eV}$ [Fig. 2(e)]. For the inverted spectrum for this class, $\delta$ again approaches $90^{\circ}$ and $270^{\circ}$ with increasing $M_{e e}$ [Fig. 2(f)]. Fig. 3(a) depicts the correlation plot between $\delta$ and $M_{e e}$ for class $V E(\mathrm{IS})$. For class $V I B$, the Dirac-type phase $\delta$ approaches $0^{\circ}$ and $180^{\circ}$ with increasing $M_{e e}$ for both normal and inverted spectra [Figs. 3(b) and 3(c)]. Fig. 3(d) shows the correlation plot between $\theta_{23}$ and $\theta_{13}$ for class $V I D$ (IS) for which $\theta_{23}$ remains below maximal for the allowed ranges of other neutrino oscillation parameters. The correlation plots between $\delta$ and $M_{e e}$ for classes $V I E$ and $V I F$ for normal and inverted spectra are shown in Figs. 3(e)(NS) and 3(f)(IS). For class VIIF(NS), $M_{e e}$ is restricted to a small range 0.008-0.028 [Fig. 4(a)] and the correlation plot depicting normal spectrum for this class is shown in Fig. 4(b). For class VIIIA(IS), the atmospheric mixing angle $\theta_{23}$ remains near maximal as shown in Fig. 4(c). Fig. 4(d) shows the correlation plot of $\delta$ and $M_{e e}$ for class $V I I I B(\mathrm{NS})$ where for larger values of $M_{e e}$, the Dirac phase $\delta$ is restricted to very small ranges.

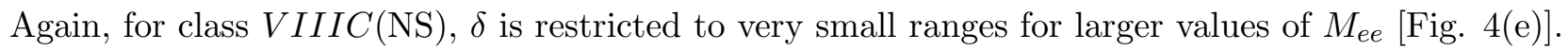
Fig. 4(f) shows the correlation plot between $\delta$ and $\alpha$ where $\alpha$ has a small allowed range near $0^{\circ}$ and $180^{\circ}$ [Fig. 4(f)]. The 2-3 interchange symmetry between classes VIIIF and VIID for NS is shown in Figs. 5(a) and 5(b). In addition to the textures for which non-zero $\theta_{13}$ is an inherent property, there are other textures namely $I A, V I I I A, I X A$ and $X A$ which forbid a vanishing $\theta_{13}$. This is because for $\theta_{13}=0$, these textures predict

$$
\left|\frac{m_{1}}{m_{2}}\right|=\left|-\cot ^{2} \theta_{12}\right|>1 \text { for the allowed range of } \theta_{12}
$$

which is contrary to the experimental observations. 

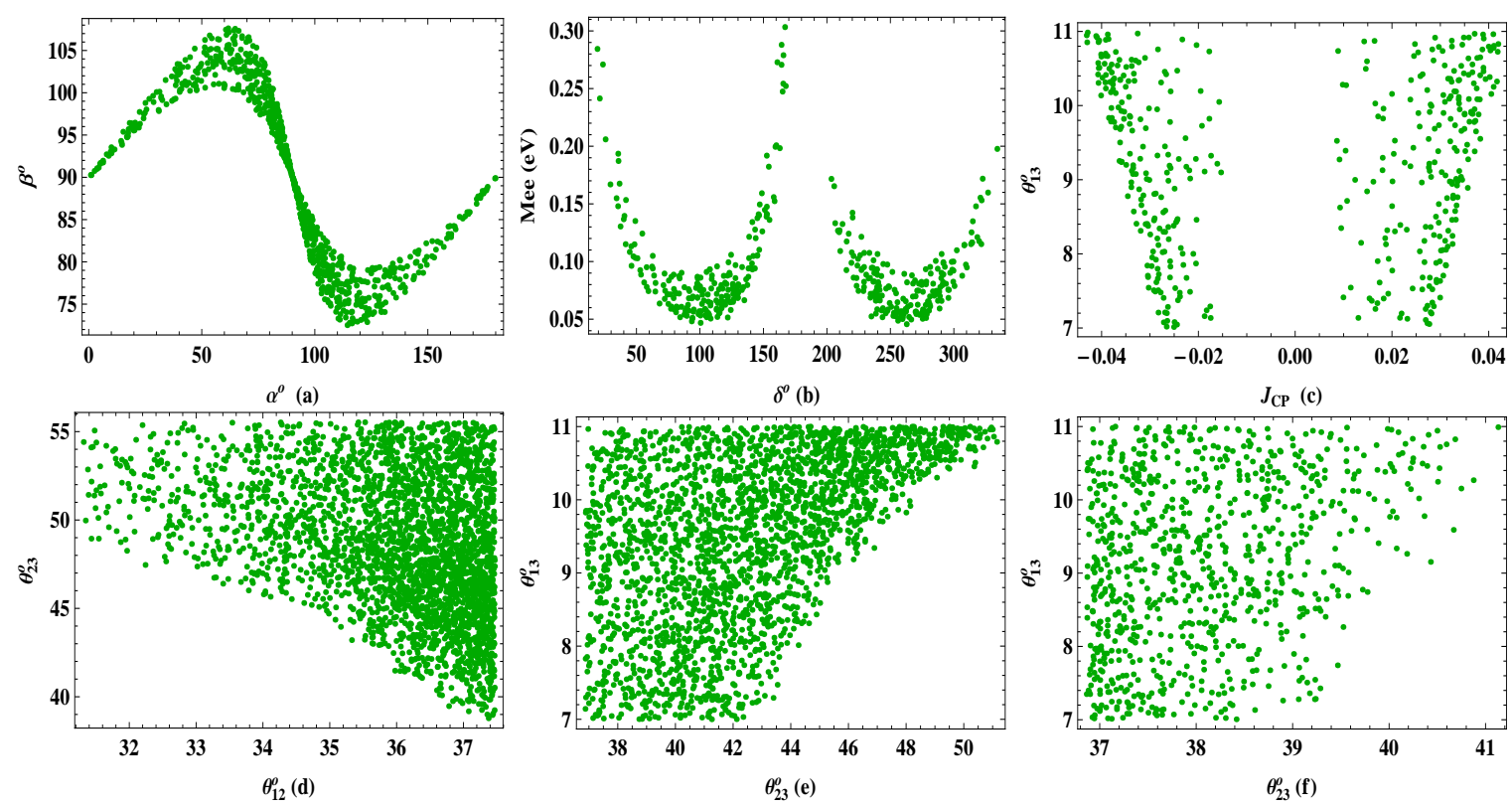

Figure 1: Correlation plots for classes $I A(\mathrm{IS})(\mathrm{a}), \operatorname{IIB}(\mathrm{NS})(\mathrm{b}, \mathrm{c}), \operatorname{IIIF}(\mathrm{NS})(\mathrm{d}), I V D(\mathrm{NS})(\mathrm{e})$ and $I V D(\mathrm{IS})(\mathrm{f})$.
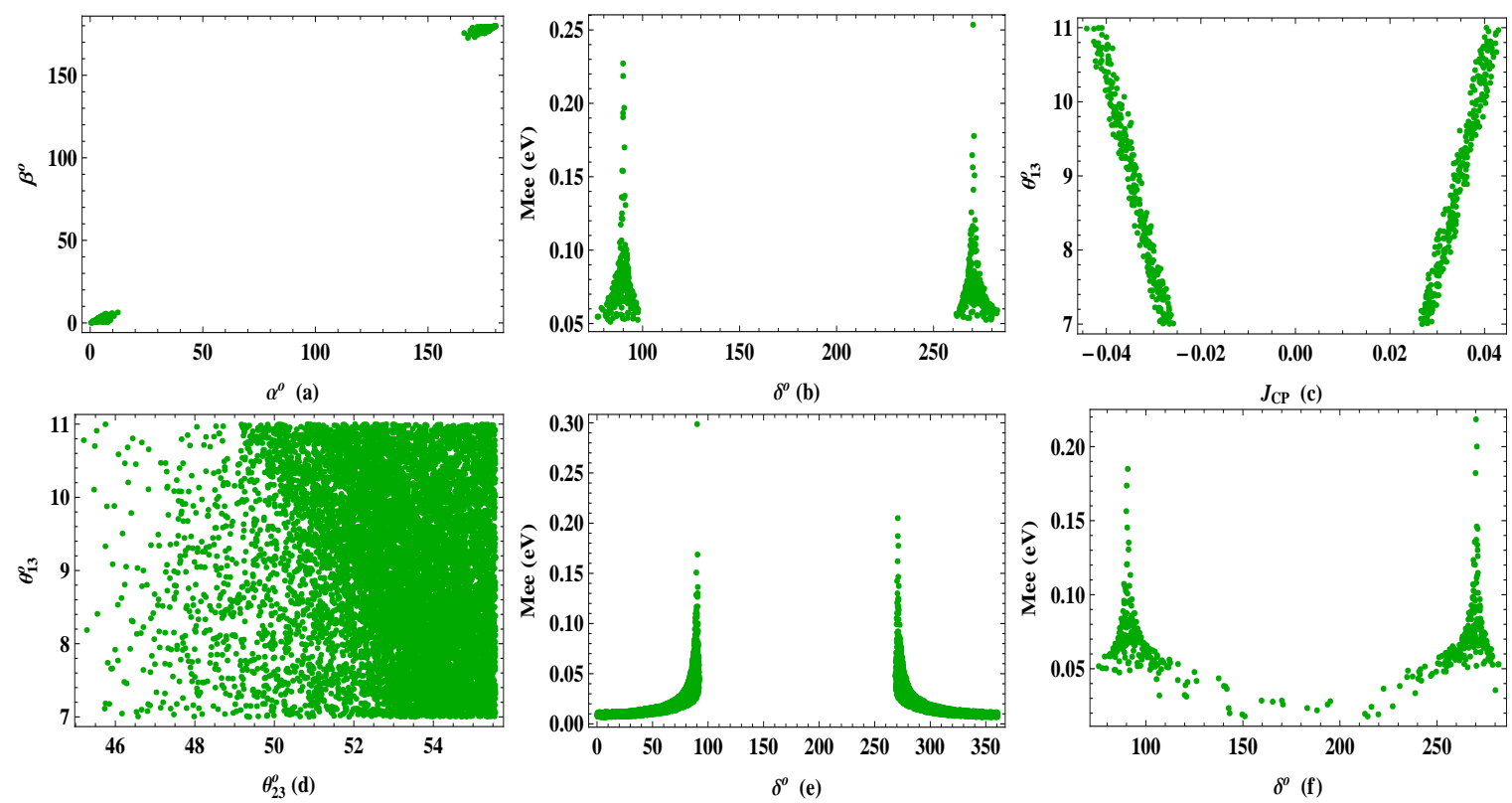

Figure 2: Correlation plots for classes $V B(\mathrm{IS})(\mathrm{a}, \mathrm{b}, \mathrm{c}), V C(\mathrm{NS})(\mathrm{d}, \mathrm{e})$ and $V C(\mathrm{IS})(\mathrm{f})$. 

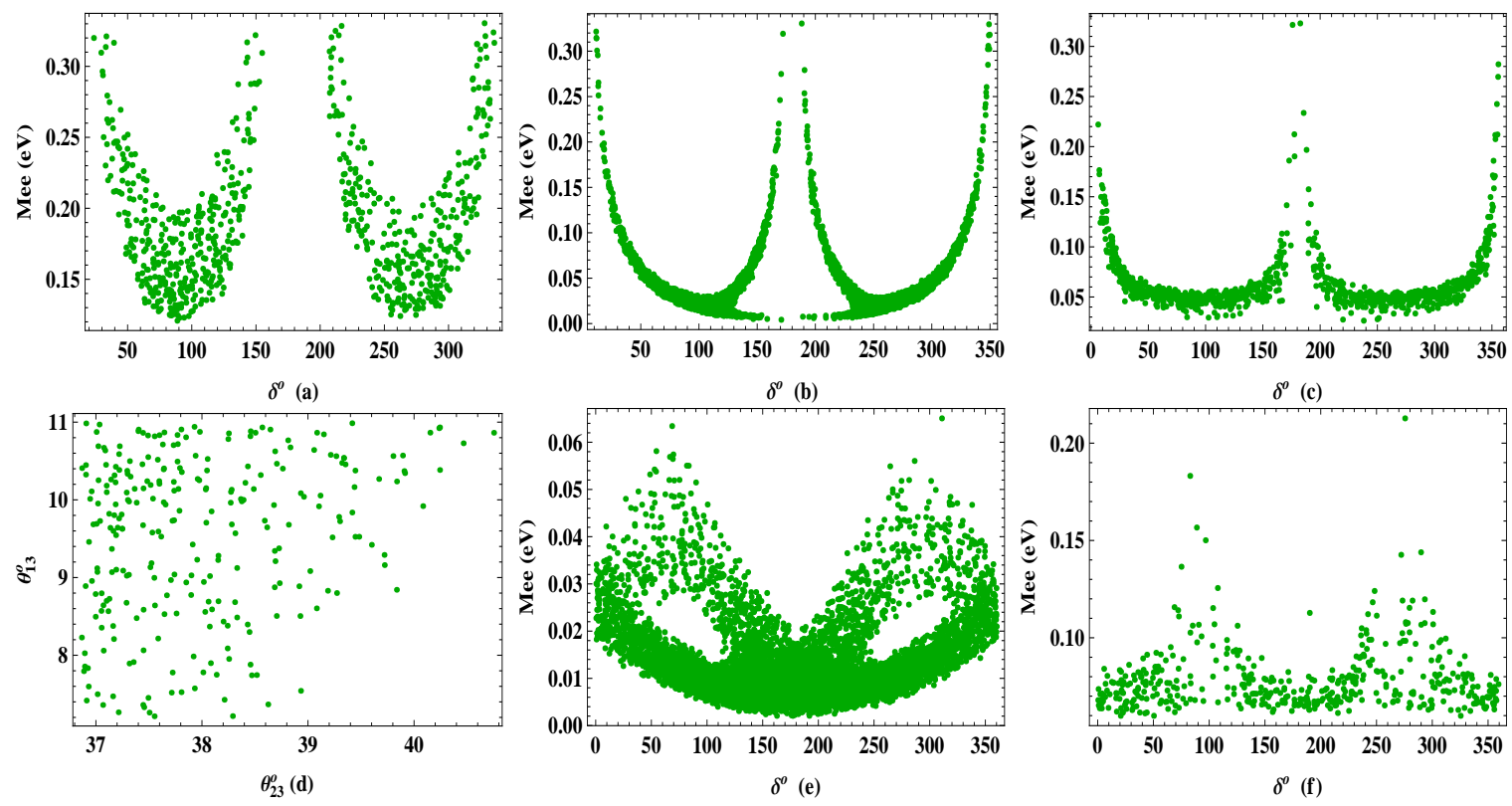

Figure 3: Correlation plots for classes $V E(\mathrm{IS})(\mathrm{a}), \quad V I B(\mathrm{NS})(\mathrm{b}), \quad V I B(\mathrm{IS})(\mathrm{c}), V I D(\mathrm{IS})(\mathrm{d})$, $V I E(\mathrm{NS})(\mathrm{e})$ and $V I F(\mathrm{IS})(\mathrm{f})$.
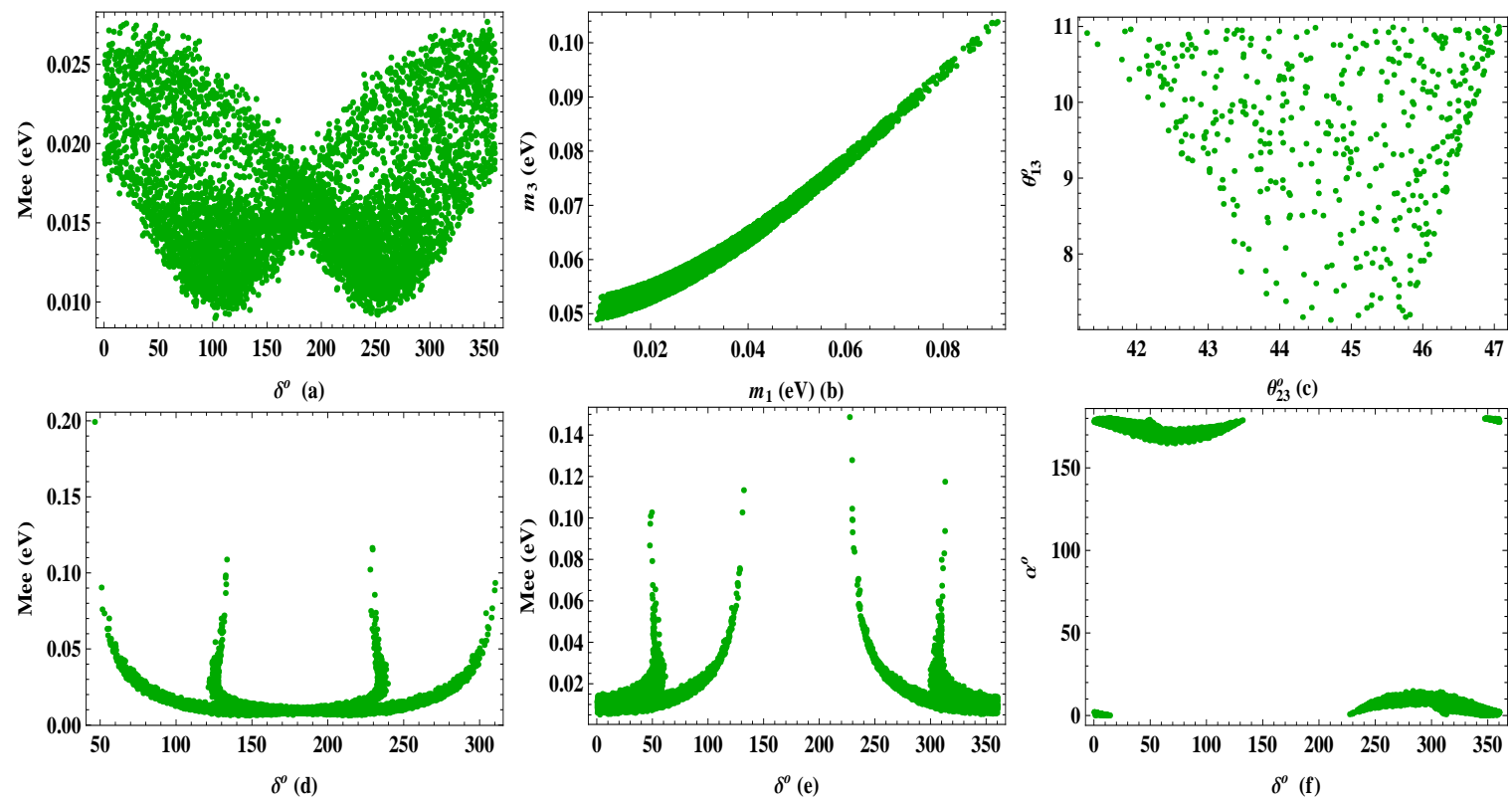

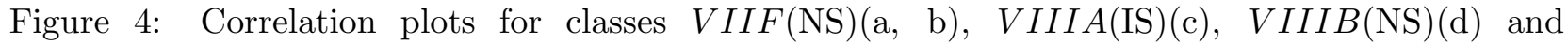
$V I I I C(\mathrm{NS})(\mathrm{e}, \mathrm{f})$. 

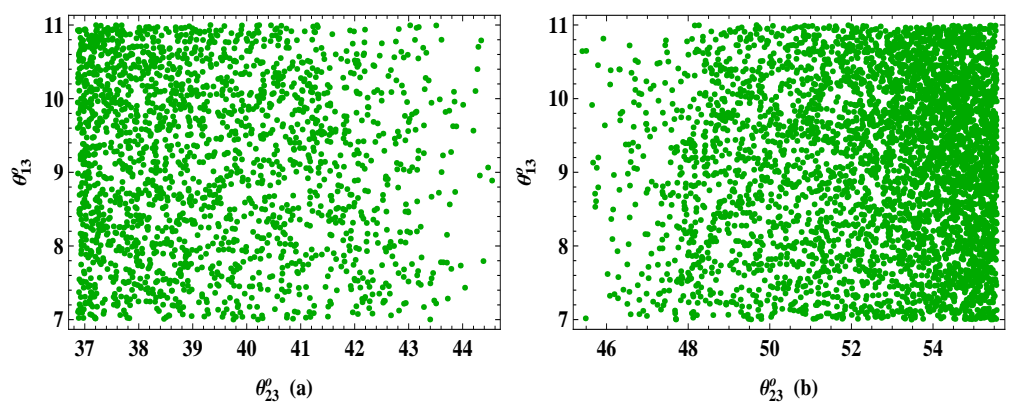

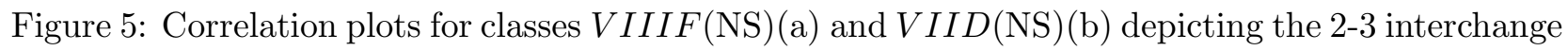
symmetry.

\section{Symmetry Realization}

We present a representative model for obtaining hybrid textures in the right-handed neutrino mass matrix $M_{R}$. Here we need one equality and one vanishing entry in $M_{R}$ in a basis where both $M_{D}$ and $M_{l}$ are diagonal with additional equality in $M_{D}$. The additional equality in $M_{D}$ is needed so that the equal elements of $M_{R}$ may propagate as equal cofactors in $M_{\nu}$. General guidelines for obtaining zero entries at any place in fermion mass matrices using Abelian discrete symmetries have been propounded in [32. In particular, all the viable cases of two zero textures in the neutrino mass matrix in flavor basis have been realized in [10, 33] considering the type-II seesaw contribution and in [34] considering both type-(I+II) seesaw contributions and using a minimal cyclic symmetry group. A few cases of hybrid textures in the effective neutrino mass matrix have been realised in [15, 35]. Here we present the symmetry realization of class $I A$ as an illustration of how the hybrid textures of $M_{\nu}^{-1}$ may be realised. For this we follow the approach of Ref. [36].

In addition to the Standard Model (SM) left-handed $S U(2)$ lepton doublets $D_{\ell L}(\ell=e, \mu, \tau)$ and the right-handed charged-lepton $S U(2)$ singlets $\ell_{R}$, we introduce three right-handed neutrinos $\nu_{\ell R}$. In the scalar sector we need three Higgs doublets $\phi_{\jmath}(\jmath=1,2,3)$, we will also need three scalar singlets. We assume the validity of the lepton number symmetries $U(1)_{L_{\ell}}$ for all dimension 4 terms in the Lagrangian. This leads to diagonal charged lepton $\left(M_{l}\right)$ and Dirac neutrino $\left(M_{D}\right)$ mass matrices. However, the dimension 3 terms are allowed to break the $L_{\ell}$ softly.

We consider the following symmetries and transformations of various fields under these symmetries:

$$
\begin{array}{rlrl}
\mathbb{Z}_{2}: & \phi_{1}, e_{R}, \nu_{\ell R} & \longrightarrow-\phi_{1},-e_{R},-\nu_{\ell R} & \\
\mathbb{Z}_{4}: & D_{\mu L} \longrightarrow i D_{\tau L}, & D_{\tau L} \longrightarrow i D_{\mu L}, \\
D_{e L} & \longrightarrow-i D_{e L}, & \mu_{R} \longrightarrow i \tau_{R}, & \tau_{R} \longrightarrow i \mu_{R}, \\
e_{R} & \longrightarrow-i e_{R}, & \nu_{\mu R} \longrightarrow i \nu_{\tau R}, & \nu_{\tau R} \longrightarrow i \nu_{\mu R}, \\
\nu_{e R} & \longrightarrow-i \nu_{e R}, & &
\end{array}
$$

These symmetries lead to the following Yukawa Lagrangian:

$$
\begin{aligned}
-\mathcal{L}_{Y}= & Y_{1}\left(\bar{D}_{e L} e_{R}\right) \phi_{1}+Y_{2}\left(\bar{D}_{\mu L} \mu_{R}+\bar{D}_{\tau L} \tau_{R}\right) \phi_{2}+Y_{3}\left(\bar{D}_{\mu L} \mu_{R}-\bar{D}_{\tau L} \tau_{R}\right) \phi_{3}+Y_{4}\left(\bar{D}_{e L} \nu_{e R}\right) \tilde{\phi}_{1} \\
& +Y_{5}\left(\bar{D}_{\mu L} \nu_{\mu R}+\bar{D}_{\tau L} \nu_{\tau R}\right) \tilde{\phi}_{1}+\text { H. c. }
\end{aligned}
$$


where $\tilde{\phi}_{1}=i \tau_{2} \phi_{1}^{*}$. When the Higgs fields $\left(\phi_{\jmath}\right)$ acquire non-zero vacuum expectation values (VEVs), we have the charged lepton mass matrix $M_{l}$ and the Dirac neutrino mass matrix $M_{D}$ of the following form:

$$
\begin{aligned}
M_{l} & =\operatorname{diag}\left(e^{i \phi_{e}} m_{e}, e^{i \phi_{\mu}} m_{\mu}, e^{i \phi_{\tau}} m_{\tau}\right) \\
M_{D} & =\operatorname{diag}(x, y, y) .
\end{aligned}
$$

where $m_{e}=\left|Y_{1}\left\langle\phi_{1}\right\rangle_{o}\right|, m_{\mu}=\left|Y_{2}\left\langle\phi_{2}\right\rangle_{o}+Y_{3}\left\langle\phi_{3}\right\rangle_{o}\right|, m_{\tau}=\left|Y_{2}\left\langle\phi_{2}\right\rangle_{o}-Y_{3}\left\langle\phi_{3}\right\rangle_{o}\right|, x=Y_{4}\left\langle\phi_{1}^{*}\right\rangle_{o}$ and $y=Y_{5}\left\langle\phi_{1}^{*}\right\rangle_{o}$.

The Majorana mass terms for the right-handed neutrinos, invariant under above symmetries are

$$
\mathcal{L}_{M}=\frac{M_{1}}{2} \nu_{e R}^{T} C^{-1}\left(\nu_{\mu R}+\nu_{\tau R}\right)+\frac{M_{2}}{2}\left(\nu_{\mu R}^{T} C^{-1} \nu_{\mu R}-\nu_{\tau R}^{T} C^{-1} \nu_{\tau R}\right)+\text { H. c. }
$$

These mass terms lead to a right-handed Majorana neutrino mass matrix having the following form

$$
M_{R}=\left(\begin{array}{ccc}
0 & a & a \\
a & b & 0 \\
a & 0 & -b
\end{array}\right) .
$$

All the non-zero entries are generated through dimension 3 terms in the Lagrangian for $M_{R}$. Now we add three complex scalar singlets $\chi_{\mu \tau}, \chi_{\mu \mu}$ and $\chi_{\tau \tau}$. The lepton number $U(1)_{L_{\ell}}$ assignments of these scalar singlets are given in Table 4 . Under the action of $\mathbb{Z}_{4}, \chi_{\mu \mu} \leftrightarrow-\chi_{\tau \tau}$ and $\chi_{\mu \tau}$ remains invariant. These scalar fields generate Yukawa couplings $Y_{6}\left[\left(\nu_{\mu R}^{T} C^{-1} \nu_{\mu R}\right) \chi_{\mu \mu}+\left(\nu_{\tau R}^{T} C^{-1} \nu_{\tau R}\right) \chi_{\tau \tau}\right]+$ H.c. and $Y_{7}\left(\nu_{\mu R}^{T} C^{-1} \nu_{\tau R}\right) \chi_{\mu \tau}+$ H.c.. When the scalar singlet fields acquire non-zero VEVs one gets the desired form of $M_{R}$ for class $I A$ :

$$
M_{R}=\left(\begin{array}{ccc}
0 & a & a \\
a & c+b & d \\
a & d & c-b
\end{array}\right)
$$

\begin{tabular}{|c|c|c|c|}
\hline & $\chi_{\mu \tau}$ & $\chi_{\mu \mu}$ & $\chi_{\tau \tau}$ \\
\hline$L_{e}$ & 0 & 0 & 0 \\
\hline$L_{\mu}$ & -1 & -2 & 0 \\
\hline$L_{\tau}$ & -1 & 0 & -2 \\
\hline
\end{tabular}

Table 4: Lepton number assignments of the scalar singlets.

\section{Summary}

We presented a detailed phenomenological analysis of neutrino mass matrices having one equality between cofactors and one vanishing cofactor. Such texture structures arise via type-I seesaw mechanism when the Dirac neutrino mass matrix is diagonal with one equality between the elements and 
the right-handed Majorana neutrino mass matrix has hybrid textures. Out of the total sixty possible hybrid textures in the right-handed Majorana neutrino mass matrix, only six are disallowed by the present neutrino oscillation data. Many of the allowed textures have constrained ranges for the unknown parameter $M_{e e}$, which will be probed in many forthcoming experiments for neutrinoless double beta decay. Some of the allowed textures have predictions for the Dirac-type CP-violating phase " $\delta$ ". Many textures also predict interesting correlations between $M_{e e}$ and $\delta$. In addition, there are predictions for the quadrant of the atmospheric mixing angle and we have compiled this information in Table 3. To show how such texture structures can be derived, we presented a flavor model for class $I A$ using discrete flavor symmetries. At present, most of the hybrid textures of the inverse neutrino mass matrix can accommodate the available experimental data. Since most of the textures studied in this analysis have predictions for one or more presently unknown neutrino parameters, experimental results on these neutrino parameters such as the quadrant of $\theta_{23}$, the value of Dirac-type CP-violating phase and the magnitude of the effective Majorana mass $M_{e e}$ will help in deciding the viability of these textures in explaining neutrino masses and mixings.

Note: After the completion of this work a similar analysis with somewhat different results came to our notice [37.

\section{Acknowledgements}

R. R. G. acknowledges the financial support provided by the Council for Scientific and Industrial Research (CSIR), Government of India.

\section{References}

[1] M. H. Ahn et al., [K2K Collaboration], Phys. Rev. Lett. 90, 041801 (2003), hep-ex/0212007; Y. Fukuda et al., [Super-Kamiokande Collaboration], Phys. Rev. Lett. 81, 1562 (1998), hep-ex/9807003; K. Eguchi et al., [KamLAND Collaboration], Phys. Rev. Lett. 90, 021802 (2003), hep-ex/0212021; T. Araki et al., [KamLAND Collaboration], Phys. Rev. Lett. 94, 081801 (2005), hep-ex/0406035; S. Abe et al., [KamLAND Collaboration], Phys. Rev. Lett. 100, 221803 (2008), arXiv:0801.4589 [hep-ex]; C. Arpesella et al., [Borexino Collaboration], Phys. Lett. B 658, 101 (2008), arXiv:0708.2251 [astro-ph]; B. T. Cleveland et al., Astrophys. J 496, 505 (1998); J. N. Abdurashitov et al., [SAGE Collaboration], J. Exp. Theor. Phys. 95, 181 (2002), astro-ph/0204245; W. Hampel et al., [GALLEX Collaboration], Phys. Lett. B 447, 127 (1999).

[2] K. Abe et al. [T2K collaboration], Phys. Rev. Lett. 107, 041801 (2011), arXiv:1106.2822 [hep-ex].

[3] P. Adamson et al. [MINOS collaboration], Phys. Rev. Lett. 107, 181802 (2011), arXiv:1108.0015 [hep-ex].

[4] Y. Abe et al., [Double Chooz collaboration], Phys. Rev. Lett. 108, 131801 (2012), arXiv:1112.6353 [hep-ex].

[5] F. P. An et al., [Daya Bay collaboration], Phys. Rev. Lett. 108, 171803 (2012), arXiv:1203.1669 [hep-ex]. 
[6] Soo-Bong Kim, for RENO collaboration, Phys. Rev. Lett. 108, 191802 (2012), arXiv:1204.0626 [hep-ex].

[7] P. F. Harrison, D. H. Perkins and W. G. Scott, Phys. Lett. B 530, 167 (2002), hep-ph/0202074; P. F. Harrison and W. G. Scott, Phys. Lett. B 535, 163 (2002), hep-ph/0203209; Zhi-zhong Xing, Phys. Lett. B 533, 85 (2002), hep-ph/0204049.

[8] C. I. Low, R. R. Volkas, Phys. Rev. D 68, 033007 (2003), hep-ph/0305243; C. S. Lam Phys. Rev. D 74, 113004 (2006), hep-ph/0611017.

[9] Paul H. Frampton, Sheldon L. Glashow and Danny Marfatia, Phys. Lett. B 536, 79 (2002), hep-ph/0201008; Zhi-zhong Xing, Phys. Lett. B 530, 159 (2002), hep-ph/0201151; Bipin R. Desai, D. P. Roy and Alexander R. Vaucher, Mod. Phys. Lett. A 18, 1355 (2003), hep-ph/0209035; A. Merle, W. Rodejohann, Phys. Rev. D 73, 073012 (2006), hep-ph/0603111; S. Dev, Sanjeev Kumar, S. Verma and S. Gupta, Nucl. Phys. B 784, 103-117 (2007), hep-ph/0611313; S. Dev, S. Kumar, S. Verma and S. Gupta, Phys. Rev. D 76, 013002 (2007), hep-ph/0612102; M. Randhawa, G. Ahuja, M. Gupta, Phys. Lett. B 643, 175-181 (2006), hep-ph/0607074; G. Ahuja, S. Kumar, M. Randhawa, M. Gupta, S. Dev, Phys. Rev. D 76, 013006 (2007), hep-ph/0703005; S. Kumar, Phys. Rev. D 84, 077301 (2011), arXiv:1108.2137 [hep-ph]; P. O. Ludl, S. Morisi, E. Peinado, Nucl. Phys. B 857, 411 (2012), arXiv:1109.3393 [hep-ph]; D. Meloni, G. Blankenburg, Nucl. Phys. B 867, 749 (2013), arXiv:1204.2706 [hep-ph]; W. Grimus, P. O. Ludl, J. Phys. G 40, 055003 (2013), arXiv:1208.4515 [hep-ph].

[10] H. Fritzsch, Zhi-zhong Xing, S. Zhou, JHEP 1109, 083 (2011), arXiv:1108.4534 [hep-ph].

[11] L. Lavoura, Phys. Lett. B 609, 317 (2005), hep-ph/0411232; E. I. Lashin and N. Chamoun, Phys. Rev. D 78, 073002 (2008), arXiv:0708.2423 [hep-ph]; E. I. Lashin, N. Chamoun, Phys. Rev. D 80, 093004 (2009), arXiv:0909.2669 [hep-ph]; S. Dev, S. Verma, S. Gupta and R. R. Gautam, Phys. Rev. D 81, 053010 (2010), arXiv:1003.1006 [hep-ph]; S. Dev, S. Gupta and R. R. Gautam, Mod. Phys. Lett. A 26, 501-514 (2011), arXiv:1011.5587 [hep-ph]; T. Araki, J. Heeck and J. Kubo, JHEP 1207, 083 (2012), arXiv:1203.4951 [hep-ph].

[12] S. Dev, S. Gupta, R. R. Gautam and L. Singh, Phys. Lett. B 706, 168 (2011), arXiv:1111.1300 [hep-ph].

[13] S. Kaneko, H. Sawanaka and M. Tanimoto, JHEP 0508, 073 (2005), hep-ph/0504074.

[14] S. Dev, S. Verma and S. Gupta, Phys. Lett. B 687, 53-56 (2010), arXiv:0909.3182 [hep-ph].

[15] Ji-Yuan Liu, Shun Zhou, Phys. Rev. D 87, 093010 (2013), arXiv:1304.2334 [hep-ph]

[16] S. Dev, S. Gupta and R. R. Gautam, Phys. Rev. D 82, 073015 (2010) arXiv:1009.5501 [hep-ph].

[17] P. Minkowski, Phys. Lett. B 67, 421 (1977); T. Yanagida, Proceedings of the Workshop on the Unified Theory and the Baryon Number in the Universe (O. Sawada and A. Sugamoto, eds.), KEK, Tsukuba, Japan, 1979, p. 95: M. Gell-Mann, P. Ramond, and R. Slansky, Complex spinors and unified theories in supergravity (P. Van Nieuwenhuizen and D. Z. Freedman, eds.), North Holland, Amsterdam, 1979, p.315; R. N. Mohapatra and G. Senjanovic, Phys. Rev. Lett. 44, 912 (1980). 
[18] S. Dev, R. R. Gautam and Lal Singh Phys. Rev. D 87, 073011 (2013) arXiv:1303.3092 [hep-ph].

[19] G. L. Fogli et al., Prog. Part. Nucl. Phys. 57, 742 (2006), hep-ph/0506083.

[20] B. Pontecorvo, Zh. Eksp. Teor. Fiz. 33, 549 (1957) [Sov. Phys. JETP 6429 (1957)].

[21] C. Jarlskog, Phys. Rev. Lett. 55, 1039 (1985).

[22] M. Tortola, J. W. F. Valle and D. Vanegas, Phys. Rev. D 86, 073012 (2012), arXiv:1205.4018 [hep-ph].

[23] C. Arnaboldi et al., [CUORICINO collaboration], Phys. Lett. B 584, 260 (2004).

[24] C. Arnaboldi et al., Nucl. Instrum. Methods Phys. Res., Sect. A 518, 775 (2004).

[25] I. Abt et al., [GERDA collaboration] hep-ex/ 0404039 .

[26] R. Gaitskell et al. [Majorana Collaboration] nucl-ex/0311013.

[27] A. S. Barabash [NEMO Collaboration], Czech. J. Phys., 52, 567 (2002), nucl-ex/0203001.

[28] M. Danilov et al., Phys. Lett. B 480, 12 (2000), hep-ex/0002003.

[29] H. V. Klapdor- Kleingrothaus, et al., Eur. Phys. J. A 12, 147 (2001), hep-ph/0103062.

[30] W. Rodejohann, Int. J. Mod. Phys. E, 20, 1833 (2011), arXiv:1106.1334 [hep-ph]

[31] P. A. R. Ade et al. [Planck Collaboration], arXiv:1303.5076] [astro-ph]

[32] W. Grimus, A. S. Joshipura, L.Lavoura and M. Tanimoto, Eur. Phys. J. C 36, 227 (2004), hep-ph/0405016.

[33] Walter Grimus, Luis Lavoura, J. Phys. G 31, 693-702 (2005), hep-ph/0412283.

[34] S. Dev, Shivani Gupta and R. R. Gautam, Phys. Lett. B 701, 605-608 (2011), arXiv:1106.3451 [hep-ph].

[35] M. Frigerio, S. Kaneko, E. Ma and M. Tanimoto Phys.Rev. D 71, 011901 (2005), hep-ph/0409187.

[36] W. Grimus, S. Kaneko, L. Lavoura, H. Sawanaka and M. Tanimoto, JHEP 01, 110 (2006), hep-ph/0510326.

[37] Weijian Wang, arXiv:1306.3556 [hep-ph]. 